\title{
Measurement of $t$-channel single top quark production in pp collisions
}

\author{
Francesco Fabozzi* \\ (on behalf of the CMS collaboration) \\ INFN-Napoli \& Università della Basilicata \\ E-mail: Francesco.Fabozzileern.ch
}

\begin{abstract}
Measurements of $t$-channel single top quark production in proton-proton collisions at the LHC at center-of-mass energies of 7 and $8 \mathrm{TeV}$, using data collected with the CMS experiment during the years 2011 and 2012, are presented. The analyses consider final states where the $\mathrm{W}$ boson from the top quark decays into electron-neutrino or muon-neutrino, and makes use of kinematic characteristics of electroweak single top production for the separation of signal from backgrounds using multivariate methods. The results are compared with the most precise Standard Model theory predictions. Measurements of top/anti-top cross section ratio and of various differential single top quark production cross sections are also presented.
\end{abstract}

The European Physical Society Conference on High Energy Physics 22-29 July 2015

Vienna, Austria

${ }^{*}$ Speaker. 


\section{Introduction}

Single top quark production at the LHC proton-proton (pp) collider [1] proceeds via three main processes: $t$ channel, $s$ channel, and associated production with a $\mathrm{W}$ boson. All of them are characterized by the presence of at least one Wtb electroweak interaction vertex, and therefore measurements of these processes allow to determine the $\left|V_{\mathrm{tb}}\right|$ element of the Cabibbo-KobayashiMaskawa (CKM) matrix. The $t$ channel has the highest cross section: at the LHC, the Standard Model (SM) prediction is about $66 \mathrm{pb}$ for $\sqrt{s}=7 \mathrm{TeV}$ and about $87 \mathrm{pb}$ for $\sqrt{s}=8 \mathrm{TeV}$ [朋. In addition, at the LHC the production mechanism depends on the quark flavour content of the proton, giving rise to an asymmetry between top and anti-top production, and therefore the measurement of the top/anti-top cross section ratio $\left(\mathrm{R}_{t-c h}\right)$ provides a constraint to the quark PDFs of the proton. The $\mathrm{R}_{t-c h}$ ratio is also sensitive to new physics arising from anomalous $\mathrm{Wtb}$ couplings or contributions from Flavour Changing Neutral Current (FCNC) processes.

In this report we review the results of the measurements of $t$-channel single top quark production performed at the CMS experiment [3] at the LHC, after having outlined the main features of the analysis strategy. The measurements are based on data sets collected during the Run 1 of the LHC, at center-of-mass energies of 7 and $8 \mathrm{TeV}$.

\section{Selection and reconstruction of $t$-channel single top events}

Single top events from $t$-channel production are reconstructed in leptonic final states, where the lepton can be a muon or an electron. Signal events are characterized by a high $p_{\mathrm{T}}$ lepton, a jet from the hadronization of the b-quark produced in the top quark decay (b-jet), and missing energy in the detector due to unreconstructed neutrino. Two additional jets, recoiling against the top quark, are also present in the final state. One jet comes from the hadronization of a light flavour quark, which tends to be emitted in the forward region. The other one is a b-jet which is expected to have low $p_{\mathrm{T}}$ and to be broadly distributed in pseudorapidity, and therefore it is likely out of the detector acceptance. The main sources of background events are $t \bar{t}, \mathrm{~W}+$ jets, and multijet events from QCD processes.

The selection criteria of signal events are similar for 7 and $8 \mathrm{TeV}$ analyses [4. 5]. Events must have exactly one identified and isolated muon or electron and at least 2 reconstructed jets, all above a certain $p_{\mathrm{T}}$ threshold. In order to identify jets from the hadronization of b-quarks, a b-tagging algorithm is employed, which is based on the 3D impact parameter of tracks associated to the jet. Selected events are then classified into independent event categories for final analysis. An event belongs to a $n$-jets $m$-tags (njmt) category if there are $n$ reconstructed jets which pass the $p_{\mathrm{T}}$ threshold, and $m$ jets of them are identified as b-jets. The $2 \mathrm{j} 1 \mathrm{t}$ category is enriched in signal $t$-channel events and it is used for signal extraction.

In order to enhance the signal component in the sample, for each event a top quark candidate is reconstructed from the kinematics of the lepton, the missing transverse momentum (in the hypothesis that it is entirely due to the undetected neutrino), and the b-jet of the event. The longitudinal component of the neutrino momentum is estimated by imposing a $\mathrm{W}$ mass constraint to the kinematics of the system formed by the lepton and the missing momentum. Only events in which the top candidate invariant mass is in the interval $[130 \mathrm{GeV}, 220 \mathrm{GeV}]$ (signal region) are selected for 
final signal extraction. Additional rejection of the QCD background is obtained by a requirement on the $\mathrm{W}$ boson transverse mass in the muon channel and a cut on the missing transverse energy in the electron channel.

Several strategies are exploited in order to extract the signal yield. One approach, employed in both the 7 and $8 \mathrm{TeV}$ analyses, is based on a maximum-likelihood fit to the distribution of the absolute value of the pseudorapidity of the non b-jet of the event $\left(\left|\eta_{j^{\prime}}\right|\right)$. Signal events tend to populate the highest part of the $\left|\eta_{j^{\prime}}\right|$ spectrum. In the $7 \mathrm{TeV}$ analysis two alternative approaches, one based on a neural network (NN) discriminant and the other one based on a boosted decision trees (BDT) discriminant, are also exploited in order to increase the separation of signal from background events. The signal yield is then extracted by a maximum-likelihood fit to the NN or BDT data distributions.

Data driven methods are implemented to characterize the distributions of the main backgrounds. Events belonging to the $3 \mathrm{j} 2 \mathrm{t}$ category are enriched in $t \bar{t}$ events and are employed as a data control sample for this source of background. Events in the sidebands of the top candidate invariant mass signal region are employed to characterize $\mathrm{W}+$ jets events, after the subtraction of the other background components. QCD background events are studied with a data control sample obtained by inverting the lepton isolation requirement.

\section{Inclusive cross section measurements}

The inclusive cross section at $7 \mathrm{TeV}$ [ 4 has been measured using a data sample equivalent to an integrated luminosity of $1.17 \mathrm{fb}^{-1}$ (muon channel) and $1.56 \mathrm{fb}^{-1}$ (electron channel). The combination of the results from the three strategies for signal extraction described above gives $\sigma_{t-c h}(7 \mathrm{TeV})=67.2 \pm 6.1($ stat + syst $) \mathrm{pb}$. The inclusive cross section at $8 \mathrm{TeV}$ [5] has been measured using a data sample equivalent to an integrated luminosity of $19.7 \mathrm{fb}^{-1}$, and the obtained result is $\sigma_{t-c h}(8 \mathrm{TeV})=83.6 \pm 2.3$ (stat) \pm 7.4 (syst) $\mathrm{pb}$. The main contributions to the systematic error on the inclusive measurements come from experimental uncertainties on the jet energy scale and resolution, and from theoretical uncertainties on the modeling of the $t$-channel signal. Figure 1 illustrates the good agreement between the measured cross sections at 7 and $8 \mathrm{TeV}$ and the theoretical expectations based on the SM. The ratio of cross sections at 8 and $7 \mathrm{TeV}$ is measured to be $\mathrm{R}_{8 / 7}=1.24 \pm 0.08$ (stat) \pm 0.12 (syst), again in agreement with the SM.

At $8 \mathrm{TeV}$ the cross sections for top and anti-top production have been also measured individually. The results are $\sigma_{\mathrm{t}}=53.8 \pm 1.5$ (stat) \pm 4.4 (syst) pb, and $\sigma_{\overline{\mathrm{t}}}=27.6 \pm 1.3$ (stat) \pm 3.7 (syst) pb, giving a top/anti-top cross section ratio $\mathrm{R}_{t-c h}=1.95 \pm 0.10$ (stat) \pm 0.19 (syst). Figure 1 compares the measured $\mathrm{R}_{t-c h}$ with theory predictions based on alternative sets of PDFs. The measurement is in agreement with predictions and it will be possible to discriminate among alternative PDF sets in future measurements with higher integrated luminosity datasets.

The CKM matrix element $\left|V_{\mathrm{tb}}\right|$ is determined from the square root of the ratio of the measured cross section over the SM expectation, provided that $V_{\mathrm{tb}}>>V_{\mathrm{td}}, V_{\mathrm{ts}}$. The combination of the measurements at 7 and $8 \mathrm{TeV}$ gives $\left|f_{\mathrm{Lv}} V_{\mathrm{tb}}\right|=0.998 \pm 0.038(\exp ) \pm 0.016$ (theo), where the first error is from experimental contributions and the second one is from the theory. The $f_{\mathrm{Lv}}$ form factor parameterizes the contribution due to anomalous couplings at the $\mathrm{Wtb}$ vertex $\left(f_{\mathrm{Lv}}(\mathrm{SM})=1\right)$. Under 


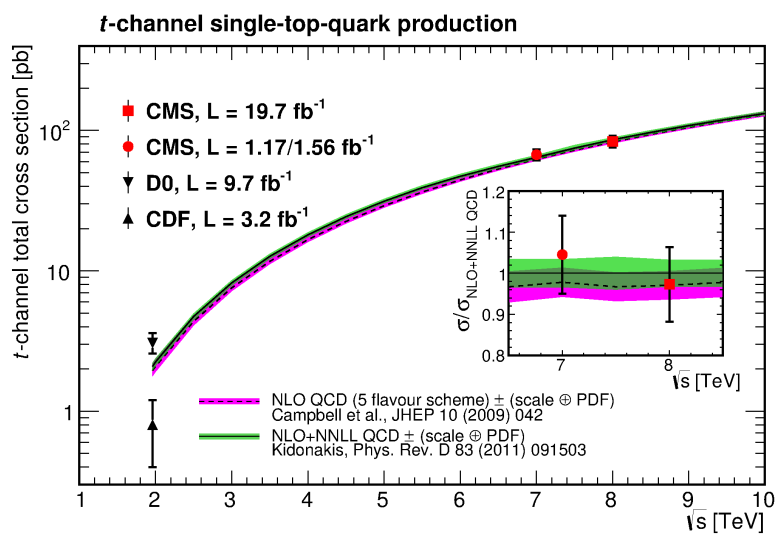

Figure 1: Single top production cross sections in the $t$ channel measured at CMS as a function of centerof-mass energy of the data set. The measurements are compared with theoretical predictions based on NLO and NLO+NNLL calculations. Measurements performed at the Tevatron proton-antiproton collider at $\sqrt{s}=1.96 \mathrm{TeV}$ are also reported.

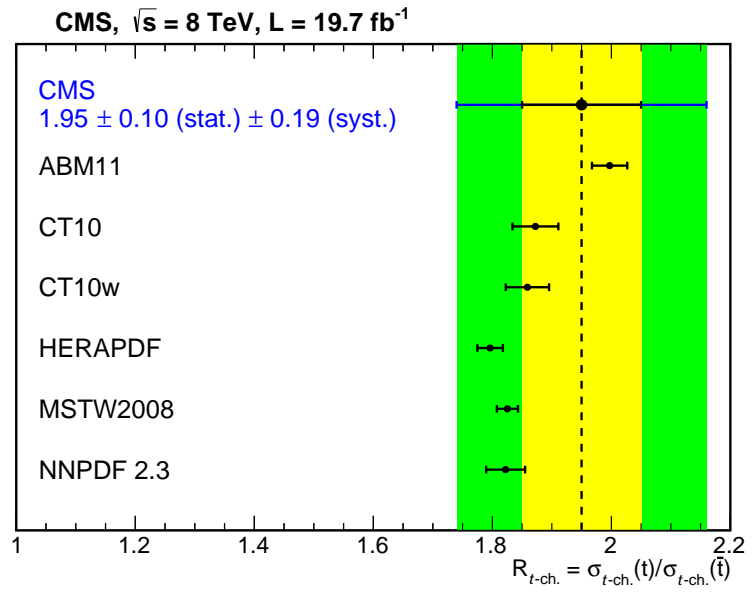

Figure 2: Measured ratio of top/anti-top cross sections in the $t$ channel compared with predictions obtained using alternative sets of PDFs.

the hypothesis of no anomalous coupling, the lower limit $\left|V_{\mathrm{tb}}\right|>0.92$ at $95 \%$ C.L. is obtained. Figure 3 summarizes the status of $\left|V_{\mathrm{tb}}\right|$ measurements at CMS based on the Run 1 data set.

\section{Differential cross section measurements}

Thanks to the large integrated luminosity of the $8 \mathrm{TeV}$ data sample, differential measurements of $t$-channel cross section have been performed, as function of the top quark $p_{\mathrm{T}}$ and rapidity [6]. In order to enhance the signal component in the data set, the analysis employes a cut on a NN discriminator built from a set of input variables, the most important ones are $\left|\eta_{j^{\prime}}\right|$ and the invariant mass 


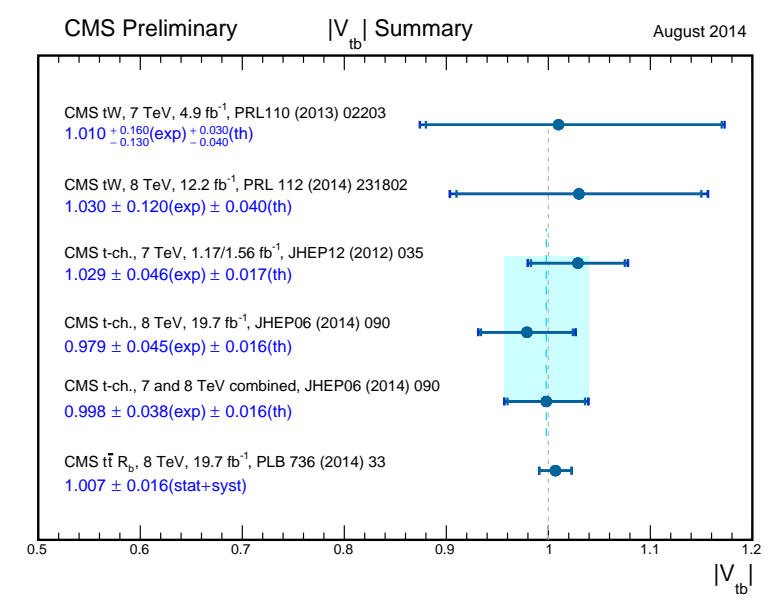

Figure 3: Summary of $\left|V_{\mathrm{tb}}\right|$ measurements at CMS based on the Run 1 data set. Individual measurements from single top events in the $\mathrm{W}$ boson associated production channel and in the $t$ channel, and from $t \bar{t}$ events are reported. The combined result from the $t$-channel measurements is also reported.

of the top quark candidate in the event. A maximum-likelihood fit to the $\mathrm{NN}$ data distribution is employed to determine the signal and the background yields. After subtraction of the background, the $p_{\mathrm{T}}$ and rapidity distributions are corrected for efficiency and resolution effects, and the unfolded spectra are obtained (Fig. \#). The results are in agreement with the predictions of different Monte Carlo generators and flavour-scheme definitions.
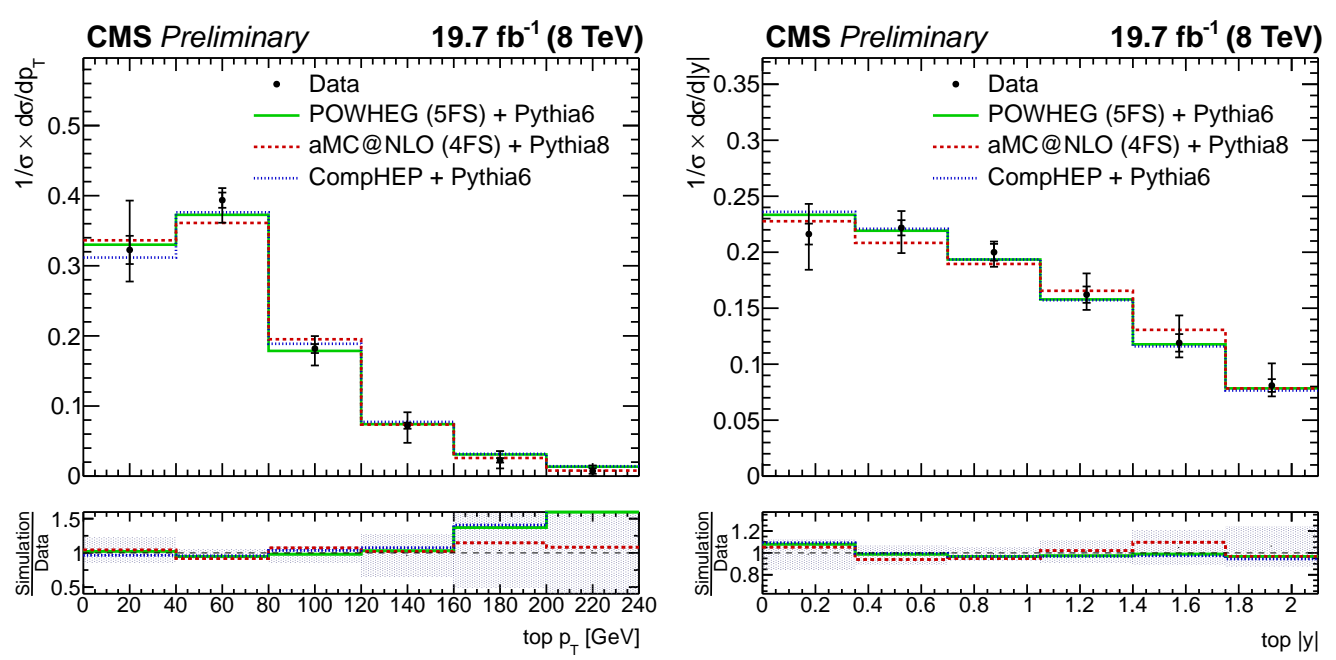

Figure 4: Unfolded $p_{\mathrm{T}}$ (left plot) and rapidity (right plot) spectra of top quark produced in single top $t$ channel events. The spectra measured in data are compared to predictions obtained from several Monte Carlo event generators: POWHEG and aMC@NLO, which are NLO generators using different flavour schemes, and CompHEP. They are interfaced with Pythia generator for hadronization and parton showering. 


\section{Summary and early inclusive measurement at $13 \mathrm{TeV}$}

Measurements of the single top quark production cross section in the $t$ channel have been performed at CMS by using pp collisions data at 7 and $8 \mathrm{TeV}$ center-of-mass energy. Measurements of inclusive production cross section have been reported, which provide also a determination of the CKM matrix element $\left|V_{\mathrm{tb}}\right|$. Preliminary measurements of differential cross section measurements at $8 \mathrm{TeV}$ have been also reported. All the results are in agreement with the SM.

Starting from June 2015 LHC has resumed operations, providing pp collisions at $\sqrt{s}=13 \mathrm{TeV}$. The expected increase of $t$-channel cross section of almost a factor of three gives the possibility of an early measurement at this unprecedented energy. Indeed in September 2015, when these proceedings were in preparation, a preliminary result based on a dataset equivalent to an integrated luminosity of $42 \mathrm{pb}^{-1}$ has been released by the CMS Collaboration 汤. The $t$-channel signal has been measured with an observed significance of 3.5 standard deviation, and the measured cross section is $\sigma_{t-c h}(13 \mathrm{TeV})=274 \pm 98$ (stat) \pm 52 (syst) \pm 33 (lumi) pb. The measurement, in agreement with the SM expectation, is still dominated by the statistical error, and the accuracy of the result is expected to improve with more data collected in Run 2.

\section{References}

[1] L. Evans and P. Bryant (editors), LHC Machine, JINST 3 (2008) S08001.

[2] N. Kidonakis, NNLL threshold resummation for top-pair and single-top production, Phys.Part.Nucl. 45 (2014) 714-722 [hep-ph/1210 . 7813].

[3] CMS Collaboration, The CMS experiment at the CERN LHC, JINST 3 (2008) S08004.

[4] CMS Collaboration, Measurement of the t-channel single top quark production cross section in pp collisions at $\sqrt{s}=7$ TeV, JHEP 12 (2012) 035, [hep-ex/1209.4533].

[5] CMS Collaboration, Measurement of the t-channel single-top-quark production cross section and of the $V_{t b} C K M$ matrix element in pp collisions at $\sqrt{s}=8 \mathrm{TeV}$, JHEP 06 (2014) 090, [hep-ex/1403.7366].

[6] CMS Collaboration, Differential measurement of the cross section of single top-quark production in the t-channel at $8 \mathrm{TeV}, \mathrm{CMS}-\mathrm{PAS}$-TOP-13-001 (2013).

[7] CMS Collaboration, Measurement of the t-channel single top-quark cross section at $13 \mathrm{TeV}$, CMS-PAS-TOP-15-004 (2015) 